14. Pauza, M.E., et al. 2004. T-cell receptor transgenic response to an endogenous polymorphic autoantigen determines susceptibility to diabetes. Diabetes. 53:978-988.

15. Lieberman, S.M., and DiLorenzo, T.P. 2003. A comprehensive guide to antibody and T-cell responses in type 1 diabetes. Tissue Antigens. 62:359-377.

16. Moriyama, H., et al. 2003. Evidence for a primary islet autoantigen (preproinsulin 1) for insulitis and diabetes in the nonobese diabetic mouse. Proc. Natl. Acad. Sci. U. S. A. 100:10376-10381.

17. Thebault-Baumont, K., et al. 2003. Acceleration of type 1 diabetes mellitus in proinsulin 2-deficient NOD mice. J. Clin. Invest. 111:851-857. doi:10.1172/ JCI200316584.

18. Jaeckel, E., Lipes, M.A., and von Boehmer, H. 2004. Recessive tolerance to preproinsulin 2 reduces but does not abolish type 1 diabetes. Nat. Immunol. 5:1028-1035

19. Kubosaki, A., et al. 2004. Targeted disruption of the IA-2beta gene causes glucose intolerance and impairs insulin secretion but does not prevent the development of diabetes in NOD mice. Diabetes. 53:1684-1691.

20. Kubosaki, A., Miura, J., and Notkins, A.L. 2004. IA2 is not required for the development of diabetes in NOD mice. Diabetologia. 47:149-150.

21. Lieberman, S.M., et al. 2003. Identification of the beta cell antigen targeted by a prevalent population of pathogenic CD8+ T cells in autoimmune diabetes. Proc. Natl. Acad. Sci. U. S. A. 100:8384-8388.

22. Robles, D.T., et al. 2002. Millennium award recipient contribution. Identification of children with early onset and high incidence of anti-islet autoantibodies. Clin. Immunol. 102:217-224.

23. Redondo, M.J., et al. 2000. DR and DQ associated protection from type 1 diabetes: comparison of DRB1*1401 and DQA1*0102-DQB1*0602. J. Clin. Endocrinol. Metab. 85:3793-3797.

24. Valdes, A.M., et al. 2005. D6S265*15 marks a DRB1*15, DQB1*0602 haplotype associated with attenuated protection from type 1 diabetes mellitus. Diabetologia. 48:2540-2543.

25. Kent, S.C., et al. 2005. Expanded T cells from pan- creatic lymph nodes of type 1 diabetic subjects recognize an insulin epitope. Nature. 435:224-228.

26. Chen, Z., Benoist, C., and Mathis, D. 2005. How defects in central tolerance impinge on a deficiency in regulatory T cells. Proc. Natl. Acad. Sci. U. S. A. 102:14735-14740.

27. Vieira, P.L., et al. 2004. IL-10-secreting regulatory T cells do not express Foxp 3 but have comparable regulatory function to naturally occurring CD4+CD25+ regulatory T cells. J. Immunol. 172:5986-5993.

28. Eisenbarth, G.S. 2005. Prediction of type 1 diabetes: the natural history of the prediabetic period. In Type 1 diabetes: molecular, cellular and clinical immunology. Barbara Davis Center for Childhood Diabetes. http://www.uchsc.edu/misc/diabetes/ oxch11.html.

29. Roep, B.O., et al. 1999. Autoreactive T cell responses in insulin-dependent (type 1) diabetes mellitus. Report of the first international workshop for standardization of T cell assays. J. Autoimmun. 13:267-282.

\title{
Bypassing complement: evolutionary lessons and future implications
}

\author{
John P. Atkinson ${ }^{1}$ and Michael M. Frank ${ }^{2}$ \\ 1Department of Medicine, Division of Rheumatology, Washington University School of Medicine, St. Louis, Missouri, USA \\ 2Department of Pediatrics, Immunology and Medicine, Duke University Medical Center, Durham, North Carolina, USA.
}

Lectins like mannan-binding protein are part of the innate immune system. They circulate in association with serine proteases. Upon binding oligosaccharides, they activate the complement cascade analogous to the more familiar but evolutionarily more recent classical pathway, which is triggered by antibody binding to antigen. In this issue of the JCI, Selander et al. developed a sensitive and specific ELISA employing Salmonella-specific sugars to assess the activity of the lectin pathway of complement activation (see the related article beginning on page 1425). This more physiologic assay system allowed the investigators to rigorously define the requirements for lectin pathway activation. Furthermore, they uncovered an unsuspected means for this pathway to reach the desired critical step of activation of the opsonin C3. These types of functional assays will eventually replace the more laborious, less physiologic, and less informative approaches currently in use to monitor complement activation.

Innate immunity suffices for most organisms on this planet. Antibodies and lymphocytes did not appear on the evolutionary scene until cartilaginous fish and sharks. The complement system is a major player in this innate immunity. The initial half of this proteolytic complement cascade accomplishes 2 goals: first, to deposit opsonic fragments derived from comple-

Nonstandard abbreviations used: MASP, MBL-associated serine protease; MBL, mannan-binding lectin.

Conflict of interest: The authors have declared that no conflict of interest exists.

Citation for this article: $J$. Clin. Invest. 116:1215-1218 (2006). doi:10.1172/JCI28622. ment components 3 and 4 (C3 and $\mathrm{C} 4$, respectively) on a target, and second, to initiate the inflammatory response through release of the $\mathrm{C} 3 \mathrm{a}$ anaphylatoxin. These 2 events occur in parallel and within minutes, making complement activation an early (if not the earliest) part of the host defense response to a microbe. Three activation pathways - classical (Figure 1), lectin (Figure 2), and alternative (Figure 3) - have been elucidated. They join at the step of C3 cleavage. Common to each pathway is the terminal half of the system, the membrane attack complex. This lytic system is initiated with the cleavage of C5 to $\mathrm{C} 5 \mathrm{a}$, another potent anaphylatoxin, and
C5b, which combines with $\mathrm{C} 6, \mathrm{C} 7, \mathrm{C} 8$, and C9 to form a pore or channel in the pathogen's cell membrane. Deficiencies in complement components predispose to infection and autoimmunity. While it is rather straightforward to account for the former, it has not been so easy for immunologists to explain the latter.

The complement system is activated by lectins, representing a preantibody means of recognizing non-self by targeting the distinct sugar profiles of microorganisms (Figure 2). Not surprisingly, parts of the lectin complement cascade were then "borrowed" by the adaptive immune system to form the antibody-triggered classi-

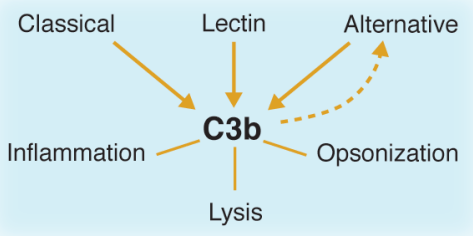

Figure 1

Three pathways of complement activation. Deposition of clusters of C3b on a target is the primary goal. The alternative pathway also serves as a feedback loop such that C3b deposition can be amplified (shown by broken line). 


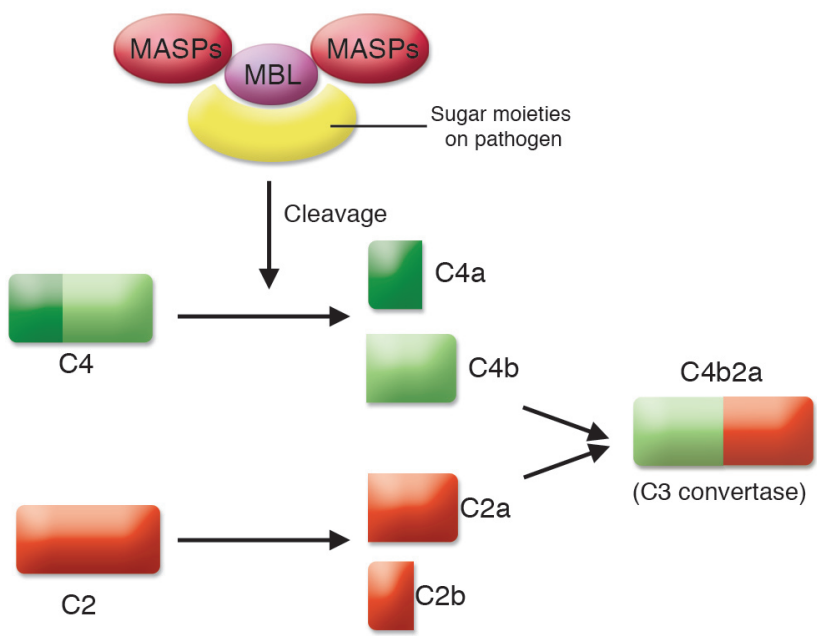

cal pathway. Complement also undergoes a continuous turnover of its key component, C3, which is the initiating event for the alternative pathway (Figure 3). Spontaneous turnover of $\mathrm{C} 3$ continuously generates $\mathrm{C} 3 \mathrm{~b}$, allowing the alternative pathway to function independently of the classical or lectin pathways. This initial C3b can also be generated via the classical or lectin pathways. On cells lacking complement inhibitors, the deposited C3 fragment $\mathrm{C} 3 \mathrm{~b}$ is the nidus for amplification via the alternative pathway.

\section{Evolution of the complement system}

The complement system is an ancient host defense pathway. As organisms became complex, the system evolved to be more efficient. The most primitive complement system probably initially consisted primarily of C3. C3 (and C4) possesses a biochemical mechanism whereby a fluidphase protein may transfer to a surface. Specifically, cleavage of C3 exposes a highly reactive thioester bond that mediates covalent attachment to hydroxyl groups on cell surfaces. Once C3b is covalently bound to a pathogen, the alternative pathway's feedback loop becomes engaged (Figure 3 ). The end result of this amplification process is deposition of large amounts of C3b, which opsonize the pathogen, and the concomitant liberation of the proinflammatory C3a anaphylatoxin.

However, this early complement system lacks a more specific means to identify appropriate targets. This void was apparently first filled with lectins such as mannan-binding lectin (MBL) or mannan-binding protein. Lectins bind repeating sugar

\section{Figure 2}

Lectin pathway. This pathway, also called the MBL pathway, is activated by the binding of $M B L$ and other lectins to sugar moieties on the surface of pathogens, which results in cleavage of $\mathrm{C} 4$ and $\mathrm{C} 2$ to produce the $\mathrm{C} 3$ convertase C4b2a. The convertase that is formed is the same as that in the classical pathway of complement activation. moieties on pathogens and then activate complement by engaging MBL-associated serine proteases (MASPs) (Figure 2). Initially, these serine proteases may have directly cleaved and thereby activated C3. To make the lectin pathway of complement activation more efficient, an intermediate step employing C4 and C2 developed. Together they form an enzyme complex C4b2a - that efficiently cleaves C3 to C3b (Figure 2). The void was next filled with the highly successful humoral immune system in which the complement activating agent antibody defines the target.

\section{Discovery of bypass pathways}

Many fits and starts and reformulations undoubtedly occurred during the evolution of the complement system. These less evolved cascades, though, are difficult to

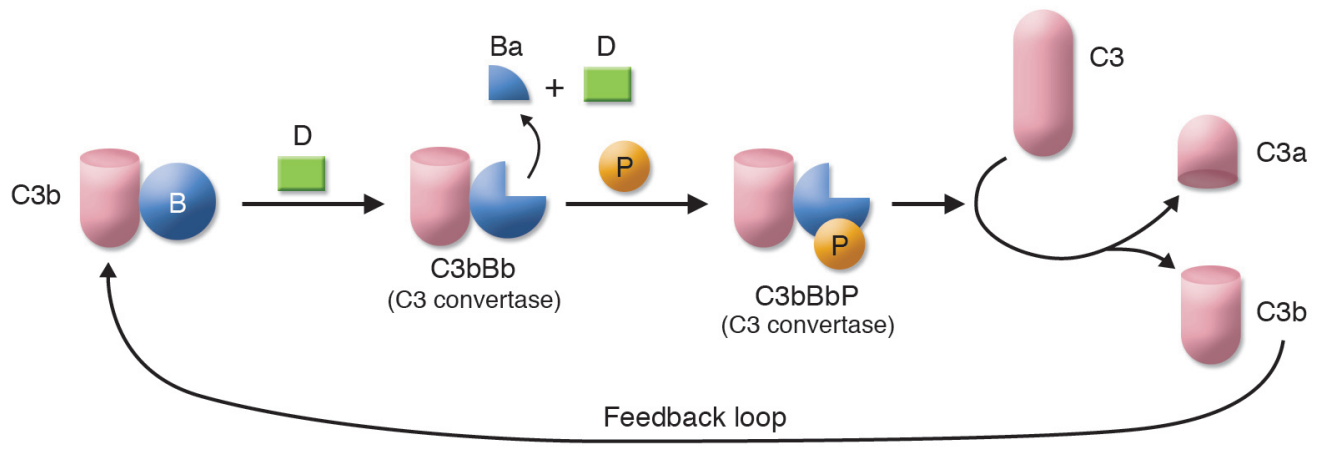

Alternative pathway

Figure 3

Feedback loop of the alternative pathway. C3b is formed by spontaneous C3 turnover in blood or generated by the lectin or classical pathways. $\mathrm{C} 3 \mathrm{~b}$ bound to a substrate binds the serine protease factor $\mathrm{B}(\mathrm{B})$. This low-affinity complex is converted by the serine protease factor $\mathrm{D}(\mathrm{D})$ to a $\mathrm{C} 3$-cleaving enzyme, $\mathrm{C} 3 \mathrm{bBb}$. This enzyme complex is stabilized by properdin (P). C3bBbP then cleaves $\mathrm{C} 3$ to $\mathrm{C} 3 \mathrm{a}$ and $\mathrm{C} 3 \mathrm{~b}$ to complete the loop and lead to amplification. 


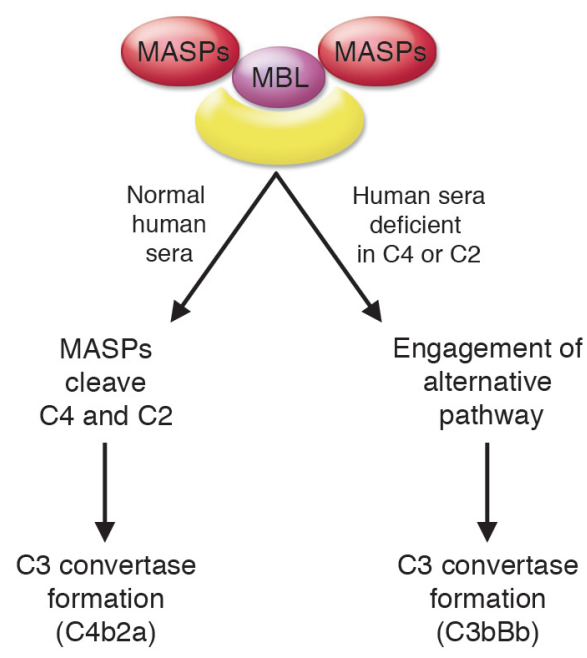

\section{Figure 4}

Lectin pathway in normal versus C2-deficient serum. In this issue of the $\mathrm{JCl}$, Selander et al. (7) examined the mechanism of complement activation by MBL in the setting of $\mathrm{C2}$ deficiency. Lectins bind sugar moieties on target pathogens and subsequently activate complement via MASPs. In normal human sera, MASPs cleave C4 and C2 to form the $\mathrm{C} 3$ convertase C4b2a. Surprisingly, Selander et al. found that in human sera from individuals deficient in $\mathrm{C} 4$ or $\mathrm{C} 2, \mathrm{MBL}$ is still capable of inducing substantial C3 deposition by engagement of the alternative pathway and formation of the $\mathrm{C} 3$ convertase $\mathrm{C} 3 \mathrm{bBb}$. This bypass pathway may be functioning in individuals with acquired or naturally occurring complement deficiencies. recognize. An obvious approach to uncover these earlier renditions of the complement system is to characterize the system in lower species. This is not so easy to achieve experimentally, however, especially in nonvertebrates, because there are over 30 distinct interacting components, receptors, and regulators to consider. A second window to evolutionary history is provided by the assessment of individuals with complement component deficiencies. Thus the first such bypass system to come to light was identified by researchers at the NIH employing C4-deficient guinea pig serum (1-3). Although they require higher quantities of sensitizing IgG or IgM than the traditional classical pathway, sheep red blood indicator cells were lysed in this serum. This C4 bypass pathway also required a C1like protein and the alternative pathway. At this juncture the lectin pathway had not yet been discovered, so the C1-like protein complex could have been a MASP. The NIH group further demonstrated that C2-deficient human serum could lyse cells under similar conditions (1-3). In other words, C4 and C2 were not necessary to lyse cells by the classical pathway.

These bypass pathways were next analyzed by Matsushita and Okada, who showed that $\mathrm{C} 4 \mathrm{~b}$ deposited on cells could facilitate alternative pathway activation in human serum lacking C2 (4). Further work on these pathways also came about because of a serendipitous observation: a patient with complete $\mathrm{C} 2$ deficiency and systemic lupus erythematosus was reported by the clinical laboratory to have a total hemolytic complement titer approximately 50\% of normal (5). As C2 is required for classical pathway activation, on multiple occasions the titer in this patient had been measured as zero. Upon further analysis, it turned out that during this recent assay, approximately 10 times the usual amount of sensitizing antibody had been employed $(5,6)$. While the total hemolytic complement titer of control (normal) serum was unchanged in the setting of these more heavily sensitized cells, serum of a C2-deficient individual was able to activate the cascade. The biochemical process that permitted this bypass pathway to be engaged was not through a hybrid classical pathway/alternative pathway convertase $(4,6)$. Instead, the formation of the alternative pathway was facilitated, probably by providing a site relatively protected from regulators.

\section{Improved assay systems to monitor complement activation}

Traditionally it has been thought that lectins activate complement through a C4and $\mathrm{C} 2$-dependent pathway analogous to the classical pathway. In this issue of the JCI, Selander et al. examined the requirements for mannan-binding protein activation of the lectin pathway (7). Of note, they employed sensitive ELISA methodology in which the lectins in the test serum bound to the O oligosaccharides of several Salmonella species. The readout was the quantitation of C3 deposition on the plate. Important features of their assay system as well as similar ones developed by others (8) are specificity for a given pathway, greater sensitivity, and use of actual pathogen material as substrates. Also, the concentrations of serum employed more closely match those in body secretions and blood. These types of assays will eventually become standard in the clinical immunology laboratory.
They provide a functional assessment, are easier to perform, and delineate more precisely and in a more physiologic manner the complement pathway(s) being activated. They will replace the current total hemolytic complement test. The widely available total hemolytic complement assay measures only the classical pathway and uses highly diluted serum and a sheep red blood cell target - none of which closely approximate the complement system interacting with a microbe in vivo.

Using an improved assay system, Selander et al. (7) demonstrate that even in the absence of $\mathrm{C} 2, \mathrm{C} 4$, or a MASP, mannanbinding protein induces substantial C3 deposition. Analogous to the results noted above relative to bypass systems in the classical cascade, these results point to a new pathway by which lectins may activate the complement pathway (Figure 4). Although this likely is not a major pathway of complement activation, it may be operative in the context of either acquired or naturally occurring complement deficiencies.

\section{Implications}

Why is this the study by Selander et al. (7) important? The primary finding is the demonstration that individuals deficient in C4 or C2 possess a "backup" or bypass complement activation system. Second, the study reaffirms the likely evolutionary history of the complement system. Third, these bypass pathways can mediate immunopathology. For example, the lysis of Giardia lamblia trophozoites (9), tissue damage in Forssman shock (10), binding of immune complexes to complement receptors $(11,12)$, and endothelial cell damage in hemolytic uremic syndrome (13) can all be shown to be 
mediated by complement bypass systems. Fourth, and probably most important, the type of assay represents the wave of the future in detecting the activity of the complement system in human disease. As these more informative detection systems (like the one used in this report) come into routine clinical use, other examples of these bypass type pathways will likely be uncovered. For human diseases, these more specific and quantitative assay systems will establish which pathway of complement activation is playing a role in disease and elucidate which one to modulate with therapeutic agents.

Finally, a word of caution is in order. These bypass pathways are often not considered by investigators attempting to define the role of the complement system in disease states. For example, C4-deficient animals are widely used to rule out a contribution of the classical pathway and/or lectin pathway in mouse models of human disease. One must be wary of such interpretations in view of bypass cascades that become operative in "deficient" states. Thus the natural maturation of an antibody response to an infectious organism (i.e., to go rapidly into antibody excess) is all that is necessary to trigger these more ancient bypass pathways. Using our current methods, such pathways are not analyzed in clinical medicine or in animal models of human disease.

Address correspondence to: John P. Atkinson, Washington University School of Medicine, 660 South Euclid Avenue, Box 8045, St. Louis, Missouri 63110, USA. Phone: (314) 362-8391; Fax: (314) 362-1366; E-mail: jatkinso@im.wustl.edu.

1. May, J.E., and Frank, M.M. 1973. A new complement-mediated cytolytic mechanism-the C1-bypass activation pathway. Proc. Natl. Acad. Sci. U. S. A. 70:649-652.

2. May, J.E., and Frank, M.M. 1973. Hemolysis of sheep erythrocytes in guinea pig serum deficient in the fourth component of complement. I. Antibody and serum requirements. J. Immunol. 111:1661-1667.

3. May, J.E., and Frank, M.M. 1973. Hemolysis of sheep erythrocytes in guinea pig serum deficient in the fourth component of complement. II. Evidence for involvement of $\mathrm{C} 1$ and components of the alternate complement pathway. J. Immunol. 111:1668-1676.

4. Matsushita, M., and Okada, H. 1986. Alternative complement pathway activation by $\mathrm{C} 4 \mathrm{~b}$ deposited during classical pathway activation. J. Immunol. 136:2994-2998.

5. Farries, T.C., Knutzen Steuer, K.L., and Atkinson, J.P. 1990. The mechanism of activation of the alternative pathway of complement by cell-bound $\mathrm{C} 4 \mathrm{~b}$. Mol. Immunol. 27:1155-1161.
6. Knutzen-Steuer, K.L., et al. 1989. Lysis of sensitized sheep erythrocytes in human sera deficient in the second component of complement. J. Immunol. 143:2256-2261.

7. Selander, B., et al. 2006. Mannan-binding lectin activates $\mathrm{C} 3$ and the alternative complement pathway without involvement of C2. J. Clin. Invest. 116:1425-1434. doi:10.1172/JCI25982.

8. Seelen, M.A., et al. 2005. Functional analysis of the classical, alternative, and MBL pathways of the complement system: standardization and validation of a simple ELISA. J. Immunol. Methods. 296:187-198.

9. Deguchi, M., Gillin, F.D., and Gigli, I. 1987. Mechanism of killing of Giardia lamblia trophozoites by complement. J. Clin. Invest. 79:1296-1302.

10. Wagner, E., Platt, J.L., Howell, D.N., Marsh, H.C., Jr., and Frank, M.M. 1999. IgG and complementmediated tissue damage in the absence of C2: evidence of a functionally active C2-bypass pathway in a guinea pig model. J. Immunol. 163:3549-3558.

11. Traustadottir, K.H., Rafnar, B.O., Steinsson, K., Valdimarsson, H., and Erlendsson, K. 1998. Participation of factor $\mathrm{B}$ in residual immune complex red cell binding activity observed in serum from a C2-deficient systemic lupus erythematosus patient may delay the appearance of clinical symptoms. Arthritis Rheum. 41:427-434.

12. Klint, C., Gullstrand, B., Sturfelt, G., and Truedsson, L. 2000. Binding of immune complexes to erythrocyte CR1 (CD35): difference in requirement of classical pathway components and indication of alternative pathway-mediated binding in C2-deficiency. Scand. J. Immunol. 52:103-108.

13. Nolin, L., et al. 1979. Possible C1q bypass loop activation in the haemolytic uraemic syndrome. Clin. Exp. Immunol. 35:107-111.

\title{
The IL-23/IL-17 axis in inflammation
}

\author{
Yoichiro Iwakura and Harumichi Ishigame
}

Center for Experimental Medicine, Institute of Medical Science, University of Tokyo, Tokyo, Japan.

\begin{abstract}
IL-23 induces the differentiation of naive $\mathrm{CD} 4^{+} \mathrm{T}$ cells into highly pathogenic helper T cells (Th17/Th ${ }_{\text {IL-17) }}$ ) that produce IL-17, IL-17F, IL-6, and TNF- $\alpha$, but not IFN- $\gamma$ and IL-4. Two studies in this issue of the JCI demonstrate that blocking IL-23 or its downstream factors IL-17 and IL-6, but not the IL-12/ IFN $-\gamma$ pathways, can significantly suppress disease development in animal models of inflammatory bowel disease and MS (see the related articles beginning on pages 1310 and 1317). These studies suggest that the IL-23/IL-17 pathway may be a novel therapeutic target for the treatment of chronic inflammatory diseases.
\end{abstract}

\section{Th17/Th $\mathrm{IL}-17_{17}$ is a new CD4+ helper T cell subset that produces IL-17}

Upon antigenic stimulation, naive $\mathrm{CD}^{+}$ $\mathrm{T}$ cells differentiate into 2 subsets, Th1 and Th2 cells, characterized by different cytokine production profiles and effector

Nonstandard abbreviations used: CIA, collageninduced arthritis; IBD, inflammatory bowel disease; IL-1Ra, IL-1 receptor antagonist; R, receptor.

Conflict of interest: The authors have declared that no conflict of interest exists.

Citation for this article: J. Clin. Invest. 116:1218-1222 (2006). doi:10.1172/JCI28508. functions (Figure 1). Th1 cells produce large quantities of IFN- $\gamma$ and mediate cellular immunity while Th2 cells, which are involved in humoral immunity, primarily produce IL-4, IL-5, and IL-13. IL-12, a heterodimer of the p40 and p35 subunits, induces the differentiation of naive $\mathrm{CD}^{+}$ $\mathrm{T}$ cells into IFN- $\boldsymbol{\gamma}$-producing Th1 cells through activation of STAT4. IFN- $\gamma$ signals are transduced by STAT1, which activates a downstream transcription factor, T-bet, that enhances the expression of genes specific to Th1 cells. In contrast, IL-4 induces
STAT6 activation, promoting the expression of GATA-3, a transcriptional factor essential for both IL-4 production and Th2 cell differentiation. Recently, it was reported that $\mathrm{CD}^{+} \mathrm{T}$ cells isolated from the inflamed joints of patients with Lyme disease contain a subset of IL-17-producing $\mathrm{CD}^{+} \mathrm{T}$ cells that are distinct from those producing either IL-4 or IFN- $\gamma$ (Figure 1) (1). These IL-17-producing CD4 ${ }^{+} \mathrm{T}$ cells were dubbed Th17 or Th $\mathrm{T}_{\mathrm{IL}-17}$ cells $(2-4)$.

IL-17, a proinflammatory cytokine predominantly produced by activated $\mathrm{T}$ cells, enhances $\mathrm{T}$ cell priming and stimulates fibroblasts, endothelial cells, macrophages, and epithelial cells to produce multiple proinflammatory mediators, including IL-1, IL- 6 , TNF- $\alpha$, NOS-2, metalloproteases, and chemokines, resulting in the induction of inflammation $(5,6)$. IL-17 expression is increased in patients with a variety of allergic and autoimmune diseases, such as RA, MS, inflamma- 